Sharif University of Technology
Scientia Iranica
SCIENTIA
I RAN ICA
http://scientiairanica.sharif.edu

\title{
Lattice Boltzmann solution of advection-dominated mass transport problem: A comparison
}

\author{
A.A. Hekmatzadeh ${ }^{\mathrm{a}, *}$, H. Keshavarzi ${ }^{\mathrm{a}}$, N. Talebbeydokhti ${ }^{\mathrm{b}, \mathrm{c}}$, and \\ A. Torabi Haghighid
}

a. Department of Civil and Environmental Engineering, Shiraz University of Technology, Shiraz, P.O. Box 71555-313, Iran.

b. Department of Civil and Environmental Engineering, Shiraz University, Shiraz, P.O. Box 7134851156, Iran.

c. Environmental Research and Sustainable Development Center, Shiraz University, Shiraz, Iran.

d. Water, Energy, and Environmental Engineering, Research Unit, University of Oulu, Finland, P.O. Box 4300, FI-90014.

Received 20 November 2017; received in revised form 10 February 2018; accepted 21 May 2018

\author{
KEYWORDS \\ Lattice Boltzmann \\ method; \\ Grid Peclet number; \\ Crank-Nicolson; \\ Artificial oscillations; \\ Multiple relaxation \\ time; \\ Equilibrium \\ distribution function.
}

\begin{abstract}
This article addresses the abilities and limitations of the Lattice Boltzmann (LB) method in solving advection-dominated mass transport problems. Several schemes of the LB method, including D2Q4, D2Q5, and D2Q9, were assessed in the simulation of two-dimensional advection-dispersion equations. The concepts of Single Relaxation Time (SRT), Multiple Relaxation Time (MRT), and linear and quadratic Equilibrium Distribution Functions (EDF) were taken into account. The results of LB models were compared to the well-known Finite Difference (FD) solutions, including Explicit Finite Difference (EFD) and Crank-Nicolson (CN) methods. All LB models are more accurate than the aforementioned FD schemes. The results also indicate the high potency of D2Q5 SRT and D2Q9 SRT in describing advection-controlled mass transfer problems. The numerical artificial oscillations are observed when the Grid Peclet Number (GPN) is greater than 10, 25, 20, 25, and 10 regarding D2Q4 SRT, D2Q5 SRT, D2Q5 MRT, D2Q9 SRT, and D2Q9 MRT, respectively, while the corresponding GPN values obtained for the EFD and CN methods were 2 and 5, respectively. Finally, several LB models were used to satisfactorily solve a coupled system of groundwater and solute transport equations. In terms of computational time, all LB models are much faster than CN method.
\end{abstract}

(C) 2020 Sharif University of Technology. All rights reserved.

\section{Introduction}

Mass transfer is one of the most abundant phenomena in the world that affects human life. Over the years, Finite Difference (FD), finite element, and finite volume methods have been successfully applied to find the solution of mass transport phenomenon for water

\footnotetext{
*. Corresponding author. Tel./Fax: +987137277656 E-mail addresses: hekmatzadeh@sutech.ac.ir (A.A. Hekmatzadeh); h.keshavarzi@sutech.ac.ir (H. Keshavarzi); taleb@shirazu.ac.ir (N. Talebbeydokhti); ali.torabihaghighi@oulu.fi (A. Torabi Haghighi)
}

resources problems [1-5]. Recently, Lattice Boltzmann (LB) method has also been taken into account as a new numerical tool for the solution of solute transport in the groundwater resources [6-8]. This method, which initiated from the lattice gas automata, investigates a problem on a mesoscopic scale. The fundamental idea of the LB method is to provide a bridge between microscopic properties of moving particles (represented by distribution function) and macroscopic variables such as fluid velocity, potential head, and pollution concentration [9-11]. Easy programming and simulation of complicated boundary conditions are the major advantages of LB methods in comparison with traditional numerical methods [12-14]. 
Different lattice configurations for LB method have been introduced to solve differential equations including D1Q2 and D1Q3 for one-dimensional problems and D2Q4, D2Q5, and D2Q9 for two-dimensional problems [15-17]. Numerous studies have been performed to solve mass transport problems, i.e., advection-dispersion equations, using LB methods, e.g., Zhou [18], Yoshida and Nagaoka [19], Perko and Patel [20], Hosseini et al. [21], Zheng et al. [22], and Bin et al. [23]. In some of these studies, the concept of Single Relaxation Time (SRT) has been employed in the mathematical formulations [18,20-22]. Aimed at improving the accuracy of the LB solution, Two Relaxation Time (TRT) and Multiple Relaxation Time (MRT) have been applied instead of SRT [24-28]. In addition, the application of quadratic Equilibrium Distribution Functions (EDF) in lieu of linear EDF in the LB method have been explored [20,23,26,29].

All of the aforementioned works have made attempts to illustrate that their introduced LB methods are appropriate for solving an ordinary solute transport equation. However, insignificant attention has been paid to the LB solution of advection-dominated transport problems, wherein advection term plays a more significant role than dispersion term $[20,25,30]$. Artificial numerical oscillations are one of the major limitations in numerical solutions of advectioncontrolled solute transport problems [31,32]. These oscillations also originate from a large grid size. In a mass transfer problem, there may be artificial fluctuations in the time-concentration curve once the pollution is approaching its final concentration. The aforementioned over/undershoots are dissipated when the numerical solution continues. It should be noted that these oscillations are different from stability issues. Such fluctuations are even observed in stable numerical solutions such as the Crank-Nicolson (CN) FD method [32]. Although several studies have focused on the stability of LB methods, few pieces of research have been dedicated to dealing with the artificial numerical oscillations [33-38].

Considering the advection-dispersion equation coupled with flow equations, Huang et al. [39] used the LB method to solve the convection-dispersion equation combined with the Navier-Stokes relations. Liu et al. [28] employed the MRT-based LB schemes for simulating heat transfer in the porous media in conjunction with the generalized Navier-Stokes equations.

To the best of our knowledge, no condition has been found to guarantee that the LB solutions are not subject to artificial over/undershoots $[18,23,30,40]$. This implies the necessity to assess different schemes of the LB method in the description of the advective mass transport problems. Moreover, few studies are devoted to resolving the advection-dispersion equation in combination with the groundwater equation $[16,41,42]$.
Hence, it may be useful to examine the joint solution of diffusion-based groundwater and advection-dispersion equations using LB method. In addition, it is important to compare the LB methods with other welldocumented numerical techniques, such as the FD methods, in order to determine the advantages and drawbacks of each method. Several FD schemes have been introduced in the literature, which are classified into explicit and implicit groups. The upwind Explicit Finite Difference (EFD) method is a fast numerical method facing instability in high time steps. The CN method is an implicit FD technique, which is stable and free of numerical dispersion. However, it requires matrix inversion in every time step, leading to high computational time [31,32].

In this treatment, several LB methods, including D2Q4 SRT, D2Q5 SRT, D2Q9 SRT, as well as D2Q5 MRT and D2Q9 MRT, were employed to solve both ordinary and advection-dominated solute transport problems in two dimensions. Both linear and quadratic EDF were taken into account in the numerical solutions. The results of the aforementioned LB methods were compared to those of two Explicit and CrankNicolson Finite Difference (EFD and CN) methods. In terms of numerical validation, the accuracy of the aforementioned models is controlled with the analytical answer of ordinary advection-dispersion equation. Besides, results of the LB methods and FD techniques are contrasted through a joint solution of advectiondispersion equation and groundwater flow concerning a recharge dam pond.

\section{Theory}

\subsection{Governing equations}

The governing equation of the solute transport problem, also called advection-dispersion equation, in porous media is as follows [32]:

$$
\frac{\partial C}{\partial t}+u_{j} \frac{\partial C}{\partial x_{j}}=D_{j} \frac{\partial^{2} C}{\partial x_{j}^{2}},
$$

where $C$ is the solute concentration, and $D_{j}$ and $u_{j}$ represent the dispersion coefficient and interstitial velocity in $j$ direction of the global coordinate system, respectively. The velocity term in Eq. (1) can be calculated using Darcy's law [43]:

$$
u_{j}=-\frac{k_{j}}{n} \frac{d h}{d x_{j}} .
$$

Here, $n$ is the porosity, $k$ stands for the hydraulic conductivity, and $h$ is the water table head, explained by the diffusion equation, describing the groundwater flow [44] as follows:

$$
\frac{\partial h}{\partial t}=\frac{K_{j}}{S_{s}} \frac{\partial^{2} h}{\partial x_{j}^{2}} .
$$


In this equation, $S_{s}$ is the specific storage coefficient.

\subsection{LB method}

Herein, the discrete Boltzmann equation is solved instead of the physical governing equations. The LB relation with the BGK collision operator can be stated as follows [45]:

$$
f_{i}\left(x+c_{i} \Delta t, t+\Delta t\right)=f_{i}(x, t)+\frac{1}{\tau}\left[f_{i}^{e q}(x, t)-f_{i}(x, t)\right],
$$

where $\tau$ represents the SRT, $f_{i}$ is the particle distribution function along the lattice direction $i, f_{i}^{e q}$ is the EDF of the particles, and $c_{i}$ is the lattice velocity in direction $i$, defined by Eq. (5). The particle distribution function represents the characteristics of the collection of particles. It should be noted that every differential equation, including advection-dispersion and groundwater equations, has a unique relaxation time and an individual EDF. The lattice direction $i$ is illustrated in Figure 1.

$$
c_{i}=\left\{\begin{array}{l}
(0,0) \quad i=0 \\
(\cos [(i-1) \pi / 2], \sin [(i-1) \pi / 2]) c \\
\quad i=1,2,3,4 \\
(\cos [(2 i-9) \pi / 4], \sin [(2 i-9) \pi / 4]) \sqrt{2} c \\
\quad i=5,6,7,8
\end{array}\right.
$$

where $c$ is the lattice velocity $(c=\Delta x / \Delta t)$. The relaxation time for the LB formulation of advectiondispersion equation is [20]:

$$
\tau_{a d}=\frac{D}{\Delta t c_{s}^{2}}+\frac{1}{2}
$$

where $c_{s}=c / \sqrt{2}$ for D2Q4 scheme, and $c_{s}=c / \sqrt{3}$ for both D2Q5 and D2Q9 schemes. In addition, the EDF for LB relation associated with the advectiondispersion equation is stated as follows [20]:

$$
f_{i}^{e q}=w_{i} C\left[1+\frac{\vec{u} \cdot c_{i}}{c_{s}^{2}}\right],
$$

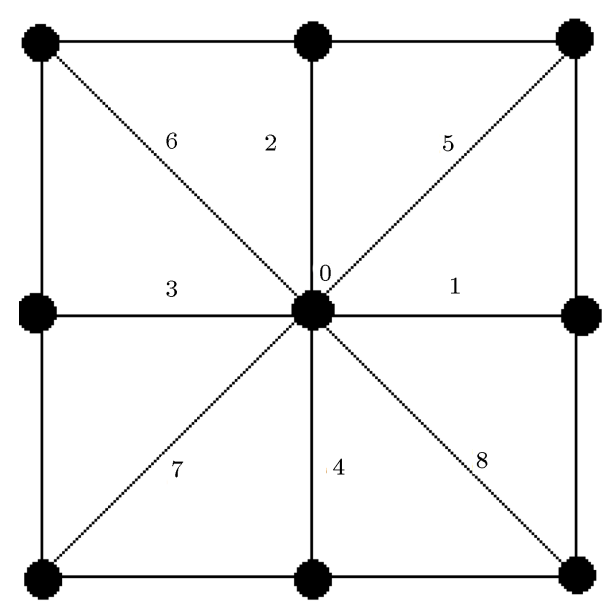

Figure 1. Directions in D2Q9 model. where $w_{i}$ is the weight coefficient in lattice direction $i$, defined by Relations (8) to (10) for different LB schemes [15]:

$$
\begin{aligned}
& \text { D2Q4 } \begin{cases}w_{i}=\frac{1}{4} & i=1,2,3,4\end{cases} \\
& \text { D2Q5 } \begin{cases}w_{i}=\frac{2}{6} & i=0 \\
w_{i}=\frac{1}{6} & i=1,2,3,4\end{cases} \\
& \text { D2Q9 } \begin{cases}w_{i}=\frac{4}{9} & i=0 \\
w_{i}=\frac{1}{9} & i=1,2,3,4 \\
w_{i}=\frac{1}{36} & i=5,6,7,8\end{cases}
\end{aligned}
$$

Finally, the solute concentration is computed by the summation of particle distribution function along all lattice directions according to Eq. (11).

$$
C=\sum_{i} f_{i}(x, t)
$$

In the case of groundwater equation (Eq. (3)), the relaxation time $\left(\tau_{g r}\right)$ and the $\operatorname{EDF}\left(g_{i}^{e q}\right)$ are respectively introduced according to Eqs. (12) and (13) [15]:

$$
\begin{aligned}
\tau_{g r} & =\frac{K}{S_{s}} \times \frac{1}{\Delta t c_{s}^{2}}+\frac{1}{2}, \\
g_{i}^{e q} & =w_{i} h .
\end{aligned}
$$

The water table, $h$, is estimated using Eq. (14):

$$
h=\sum_{i} g_{i}(x, t)
$$

In the above-mentioned LB relation (Eq. (4)), the concept of SRT has been employed, which indicates that all particles relax in their equilibrium state with the same rate. Nonetheless, the relaxation time is different in each lattice direction. Therefore, a LB relation with MRT has also been developed, stated as [45]:

$$
\begin{aligned}
f_{i}(x & \left.+c_{i} \Delta t, t+\Delta t\right)-f_{i}(x, t) \\
& =M^{-1} S\left[m^{e q}(x, t)-m(x, t)\right],
\end{aligned}
$$

where $S$ is the relaxation matrix, which is diagonal in the cases of isotropic problems. Matrix $S$ is defined by Eqs. (16) and (17) for D2Q5 and D2Q9 schemes, respectively $[27,28]$ :

$$
\begin{aligned}
& S_{\mathrm{D} 2 \mathrm{Q} 5}=\operatorname{diag}\left(\lambda_{1}, \lambda_{2}, \lambda_{3}, \lambda_{4}, \lambda_{5}\right), \\
& S_{\mathrm{D} 2 \mathrm{Q} 9}=\operatorname{diag}\left(\lambda_{1}, \lambda_{2}, \lambda_{3}, \lambda_{4}, \lambda_{5}, \lambda_{6}, \lambda_{7}, \lambda_{8}, \lambda_{9}\right),
\end{aligned}
$$

where $\lambda_{i}$ is between 0 and 2 . The relaxation times can be optimized to boost the performance of the schemes. 
Regarding D2Q5 scheme, Liu et al. (2014) proposed that $\lambda_{1}=1, \lambda_{2}=\lambda_{3}=1 / \tau_{a d}, \lambda_{4}=\lambda_{5}=1.5[28]$. However, Chai and Zhao (2014) suggested that $\lambda_{1}=0$, $\lambda_{2}=\lambda_{3}=1, \lambda_{4}=1 / \tau_{a d}, \lambda_{5}=1, \lambda_{6}=1 / \tau_{a d}, \lambda_{7}=$ $\lambda_{8}=\lambda_{9}=1$ [27]. It should be noted that the term "diag" implies diagonal matrix in the above relations.

Moreover, $m$ and $m^{e q}$ are vectors that indicate distribution functions in the momentum space $(m=$ $M f$ and $m^{e q}=M f^{e q}$ ). Additionally, $M$ is a transformation matrix that makes a connection between the distribution functions and the macroscopic moments linearly $[7,45]$. The transformation matrixes for D2Q5 and D2Q9 schemes are expressed according to Eqs. (18) and (19), respectively $[27,28]$ :

$M_{\mathrm{D} 2 \mathrm{Q} 5}=\left[\begin{array}{ccccc}1 & 1 & 1 & 1 & 1 \\ 0 & 1 & 0 & -1 & 0 \\ 0 & 0 & 1 & 0 & -1 \\ -4 & 1 & 1 & 1 & 1 \\ 0 & 1 & -1 & 1 & -1\end{array}\right]$

$M_{\mathrm{D} 2 \mathrm{Q} 9}=\left[\begin{array}{ccccccccc}1 & 1 & 1 & 1 & 1 & 1 & 1 & 1 & 1 \\ -4 & -1 & -1 & -1 & 2 & 2 & 2 & 2 & 2 \\ 4 & -2 & -2 & -2 & -2 & 1 & 1 & 1 & 1 \\ 0 & 1 & 0 & -1 & 0 & 1 & -1 & -1 & 1 \\ 0 & -2 & 0 & 2 & 0 & 1 & -1 & -1 & 1 \\ 0 & 0 & 1 & 0 & -1 & 1 & 1 & -1 & -1 \\ 0 & 0 & -2 & 0 & 2 & 1 & 1 & -1 & -1 \\ 0 & 1 & -1 & 1 & -1 & 0 & 0 & 0 & 0 \\ 0 & 0 & 0 & 0 & 0 & 1 & -1 & 1 & -1\end{array}\right]$.

It should be noted that the distribution function can also be defined by higher order velocity terms or quadratic form. In this context, the EDF for LB relation with regard to the advection-dispersion equation is as follows [25]:

$$
f_{i}^{e q}=w_{i} C\left[1+\frac{u \cdot c_{i}}{c_{s}^{2}}+\frac{\left(u \cdot c_{i}\right)^{2}}{2 c_{s}^{4}}-\frac{u^{2}}{2 c_{s}^{2}}\right] .
$$

In order to illustrate the implication of different boundary conditions, D2Q9 scheme is taken into account (see Figure 1). When the variable at the east boundary is specified (the Dirichlet boundary condition), the particle distribution functions in certain directions are obtained using Eq. (21) [15,45]:

$$
\begin{aligned}
& f_{1}=\left(w_{1}+w_{3}\right) \times C-f_{3}, \\
& f_{5}=\left(w_{5}+w_{7}\right) \times C-f_{7}, \\
& f_{8}=\left(w_{8}+w_{6}\right) \times C-f_{6},
\end{aligned}
$$

where $f_{3}, f_{6}$, and $f_{7}$ are obtained through the streaming process. In terms of the Neumann boundary condition, where the variable gradient is identified at boundaries, $f_{i}$ is estimated using Eq. (22) $[15,45]$ :

$$
\begin{aligned}
& f_{1}(n)=f_{1}(n-1), \\
& f_{5}(n)=f_{5}(n-1), \\
& f_{8}(n)=f_{8}(n-1) .
\end{aligned}
$$

In the above relation, $n$ represents the last lattice node. In the open boundary condition, the outlet variable is not known. A normal practice involves using extrapolation for the unknown distribution functions. In the east boundary condition, the following relation is applied $[15,45]$ :

$$
\begin{aligned}
& f_{1}(n)=2 \times f_{1}(n-1)-f_{1}(n-2), \\
& f_{5}(n)=2 \times f_{5}(n-1)-f_{5}(n-2), \\
& f_{8}(n)=2 \times f_{8}(n-1)-f_{8}(n-2) .
\end{aligned}
$$

\subsection{FD method}

In order to measure the precision of LB models with the traditional FD methods, both explicit and implicit finite difference techniques were employed. The general form of FD approximation of Eq. (1) is [31,32]:

$$
\begin{aligned}
& \frac{C_{i}^{n+1}-C_{i}^{n}}{\Delta t}=(1-\omega)\left\{\frac{D}{\left(\Delta x_{j}\right)^{2}}\left(C_{i+1}^{n}-2 C_{i}^{n}+C_{i-1}^{n}\right)\right. \\
& \left.-\frac{u}{\Delta x_{j}}\left[(1-\alpha) C_{i}^{n}+\alpha C_{i+1}^{n}-(1-\alpha) C_{i-1}^{n}-\alpha C_{i}^{n}\right]\right\} \\
& +\omega\left\{\frac{D}{\left(\Delta x_{j}\right)^{2}}\left(C_{i+1}^{n+1}-2 C_{i}^{n+1}+C_{i-1}^{n+1}\right)\right. \\
& -\frac{u}{\Delta x_{j}}\left[(1-\alpha) C_{i}^{n+1}+\alpha C_{i+1}^{n+1}-(1-\alpha) C_{i-1}^{n+1}\right. \\
& \left.\left.-\alpha C_{i}^{n+1}\right]\right\}
\end{aligned}
$$

where $n$ and $i$ are the time and length indices, $\omega$ and $\alpha$ denote temporal and spatial weighting factors, respectively, and $j$ represents the $x$ and $y$ directions. On the condition that $\omega=0.5$, the FD approximation (Eq. (24)) is reduced to CN method, which is an implicit scheme. In the case where $\omega=0$, the approximation is called EFD method. For the above FD models, $\alpha$ was set to 0.5 , representing the central difference in the spatial derivatives [32]. The central difference is accurate with respect to the second order, eliminating the numerical dispersion. 


\section{Examples}

\subsection{Two-dimensional solute transport problem} In order to assess different schemes of the LB method in the solution of the advection-dominated solute transport equation, a two-dimensional mass transport problem was considered at a domain size of $100 \mathrm{~m} \times$ $100 \mathrm{~m}$, where the pollution entered across $20 \mathrm{~m}$ of the left side (Figure 2). The Neumann boundary conditions were assumed for the borders of the region except for the line $\mathrm{AB}$, where the boundary condition was Dirichlet. Different values of dispersion coefficients, ranging from 0.001 to $0.05 \mathrm{~m}^{2} / \mathrm{s}$, were incorporated to consider both ordinary and advection-controlled solute transport problems. The model parameters including the dispersion coefficient and the velocity term in addition to the Grid Peclet Number $(\mathrm{GPN}=u \Delta x / D)$ are listed in Table 1.

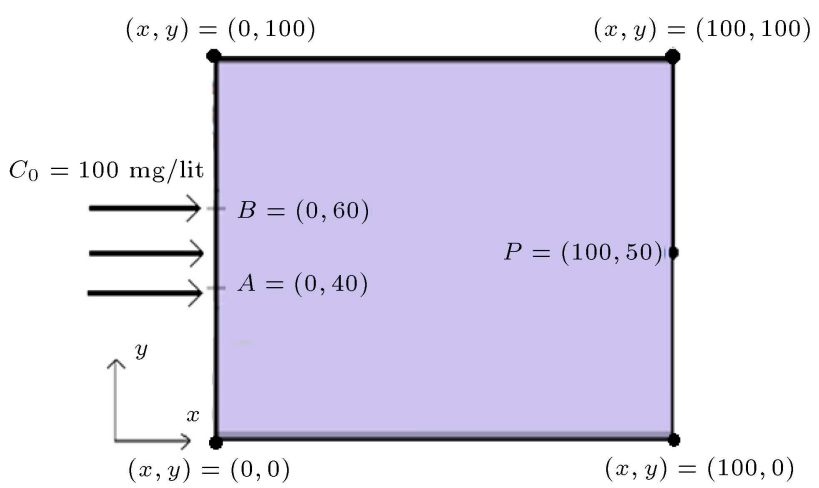

Figure 2. Schematic representation of $2 \mathrm{D}$ mass transport problem.

Table 1. Model parameters of ordinary and advective solute transport problems.

\begin{tabular}{cccccc}
\hline Models & $\begin{array}{c}\boldsymbol{D}_{\boldsymbol{x}} \\
\left(\mathbf{m}^{\mathbf{2}} / \mathbf{m i n}\right)\end{array}$ & $\begin{array}{c}\boldsymbol{u}_{\boldsymbol{x}} \\
(\mathbf{m} / \mathbf{m i n})\end{array}$ & $\begin{array}{c}\boldsymbol{\Delta} \boldsymbol{x} \\
(\mathbf{m})\end{array}$ & $\begin{array}{c}\boldsymbol{\Delta} \boldsymbol{t} \\
(\mathbf{m i n})\end{array}$ & $\mathbf{G P N}$ \\
\hline Model 1 & 0.050 & 0.05 & 1 & 0.5 & 1 \\
Model 2 & 0.0033 & 0.05 & 1 & 0.5 & 15 \\
Model 3 & 0.001 & 0.05 & 1 & 0.5 & 50 \\
Model 4 & 0.0005 & 0.05 & 1 & 0.5 & 100 \\
\hline
\end{tabular}

The advection-controlled problems may be subject to numerical artificial oscillations. Accordingly, the oscillation rate is defined by Eq. (25):

$$
\mathrm{Or}=\frac{\max (\text { Oscillation })-C_{0}}{C_{0}}
$$

where Or stands for the Oscillation Rate, and $C_{0}$ is the average value of breakthrough curve at the termination time. The error between numerical and analytical solutions is predicted using the Root Mean Square of Errors (RMSE), stated as in Eq. (26) [46].

$$
\mathrm{RMSE}=\sqrt{\frac{1}{n} \sum_{i=1}^{n}\left(C_{n u m, i}-C_{a n a, i}\right)^{2}} .
$$

In this study, several MATLAB codes were provided for the LB and FD models. It should be noted that the analytical solution to the above-mentioned solute transport problem is stated as in Eq. (27) [47]:

$$
\begin{gathered}
C(x, y, t)=\frac{C_{0} \cdot x}{4 \sqrt{\pi D_{x}}} \exp \left(\frac{V x}{2 D_{x}}\right) \\
\times \int_{\tau=0}^{\tau=t} \tau^{\frac{-3}{2}} \exp \left[-\left(\frac{V^{2}}{4 D_{x}}\right) \tau-\frac{x^{2}}{4 D_{x} \tau}\right] \\
\left\{\operatorname{erfc}\left[\frac{\left(y_{1}-y\right)}{2 \sqrt{D_{y} \tau}}\right]-\operatorname{erfc}\left[\frac{\left(y_{2}-y\right)}{\sqrt{D_{y} \tau}}\right]\right\} d \tau,
\end{gathered}
$$

where $y_{1}$ and $y_{2}$ are the transversal locations of input pollution plume (see Figure 2). The numerical solutions were verified through Eq. (24).

\subsection{Recharge dam}

In this example, the LB solution of advectiondispersion equation in a domain with variable velocity terms was examined. For this purpose, a recharge dam was assumed on a permeable river bed according to Figure 3. This is a procedure for groundwater recharge during a flood event in dry regions [48]. The

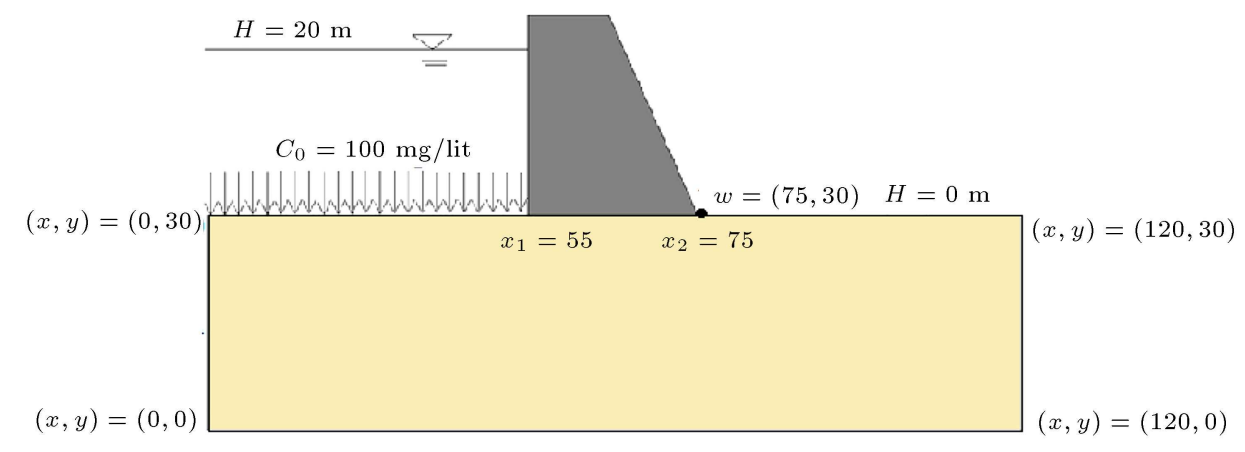

Figure 3. Schematic representation of the recharge pond problem. 
recharging water, resulting from surface runoff, is usually polluted [49]. Consequently, a coupled system of groundwater and solute transport equations was solved in two dimensions. It was assumed that the water level rose up to $20 \mathrm{~m}$ abruptly during the flood. The presumed model parameters were $K=0.002 \mathrm{~m} / \mathrm{min}$, $S_{s}=0.0001 \mathrm{~m}^{-1}, D_{x}=0.02 \mathrm{~m}^{2} / \mathrm{min}$, and $D_{y}=$ $0.02 \mathrm{~m}^{2} / \mathrm{min}$. In this problem, the velocity components are variables along both $x$ and $y$ directions, which are calculated by solving the groundwater equation (Eq. (3)). The boundary conditions of the groundwater equation are stated in Relations (28):

$$
B . C\left\{\begin{array}{l}
H=20 \mathrm{~m}, \rightarrow y=30 \mathrm{~m}, \quad 0<x<55 \mathrm{~m} \\
H=0, \rightarrow y=30 \mathrm{~m}, \quad 75 \mathrm{~m}<x<120 \mathrm{~m} \\
\frac{\partial H}{\partial y}=0, \rightarrow y=0 \\
\frac{\partial H}{\partial y}=0, \rightarrow 55 \mathrm{~m}<x<75 \mathrm{~m}, \quad y=30 \mathrm{~m} \\
\frac{\partial H}{\partial x}=0, \rightarrow x=0, \quad x=120 \mathrm{~m}, \quad 0<y<30 \mathrm{~m}
\end{array}\right.
$$

In terms of solute transport or advection-dispersion equation, a combination of Dirichlet, Neumann, and open boundary conditions was taken into consideration, as explained in Eq. (29):

$B . C\left\{\begin{array}{l}C=C_{0}, \rightarrow y=30 \mathrm{~m}, \quad 0<x<55 \mathrm{~m} \\ \frac{\partial C}{\partial y}=0, \rightarrow y=30 \mathrm{~m}, \quad 55 \mathrm{~m}<x<75 \mathrm{~m} \\ \text { open boundary } \rightarrow y=30 \mathrm{~m}, 75 \mathrm{~m}<x<120 \mathrm{~m}(29) \\ \text { open boundary } \rightarrow x=0, \quad x=120 \mathrm{~m}\end{array}\right.$

\section{Results and discussion}

\subsection{Numerical results for advection-dominated solute transport problem}

Figures 4 and 5 show the time-concentration curves predicted by the aforementioned numerical schemes for an advection-dominated transport problem at the GPN of $15 \mathrm{~m}$ and $50 \mathrm{~m}$ (Models 2 and 3 in Table 1), respectively. These figures are depicted at the middle point on the right side of the domain (point $\mathrm{P}$ in Figure 2). Accordingly, there are relatively high artificial oscillations in the FD solutions (EFD and CN), whereas LB methods comprising D2Q4 SRT, D2Q5 SRT, D2Q5 MRT, D2Q9 SRT, and D2Q9 MRT have no or low artificial oscillations. According to Figure 5, for which

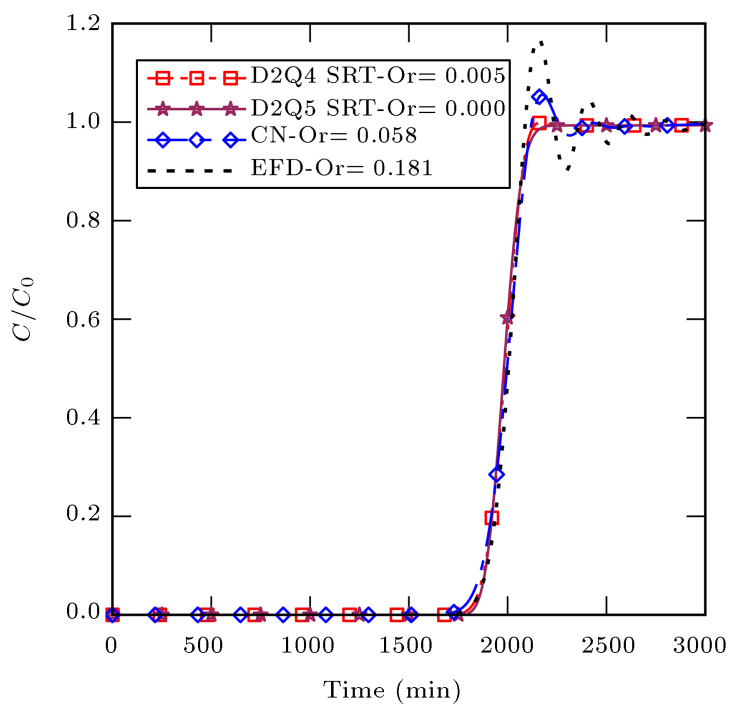

(a)

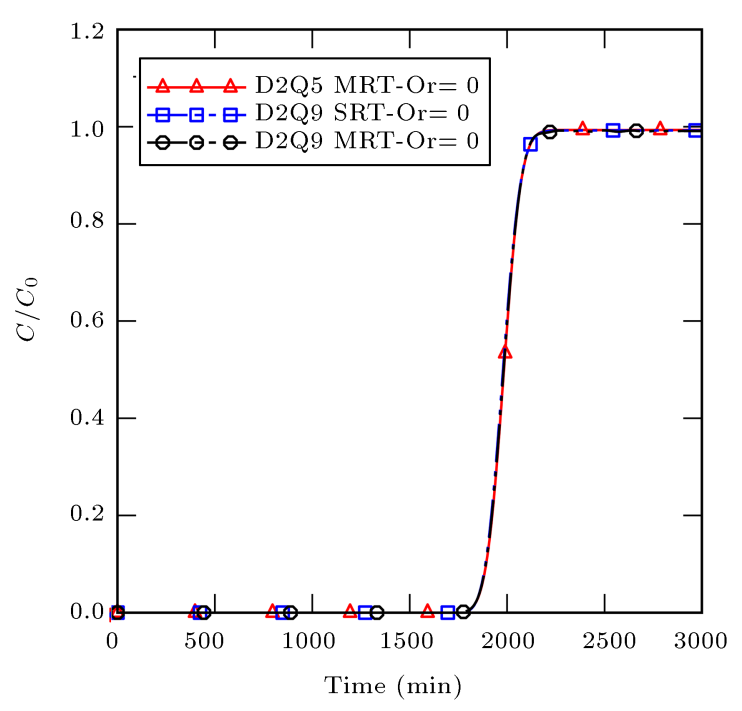

(b)

Figure 4. Comparison of numerical solutions for an advective 2D solute transport at $\mathrm{GPe}=15$ : (a) D2Q4 SRT, D2Q5 SRT, Crank-Nicolson (CN), and Explicit Finite Difference (EFD) and (b) D2Q5 MRT, D2Q9 SRT, and D2Q9 MRT.

the GPN is 50, D2Q4 SRT has the highest oscillation rate amongst the presented LB schemes. However, its maximum oscillation rate is 0.1 , which is nearly $60 \%$ of the one obtained by CN scheme. Moreover, D2Q5 SRT and D2Q9 SRT, in addition to D2Q5 MRT, undergo relatively small values of artificial undulations. Similar results are also observed according to the longitudinalconcentration curve in Figure 6. This figure indicates the concentration along the horizontal line passing through the point $\mathrm{P}$ when the time is equal to 1500 min. It should be pointed out that D2Q9 MRT has become unstable at GPN of 50 and, consequently, no solution has been achieved at this GPN. Moreover, the 


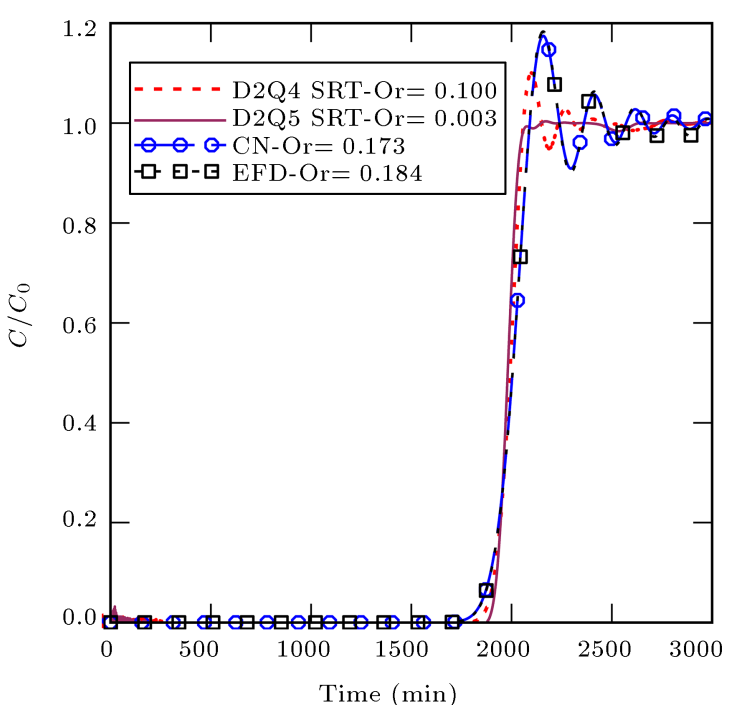

(a)

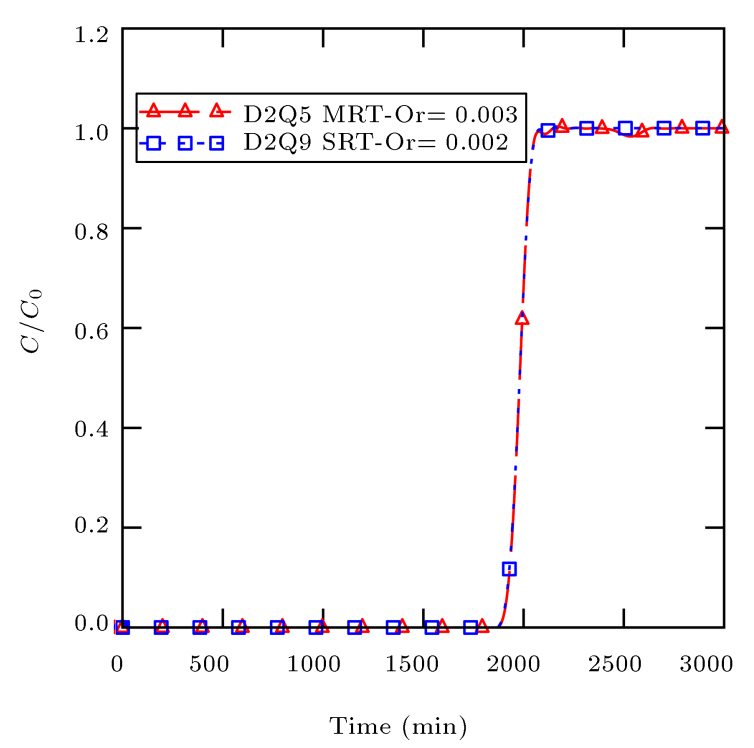

(b)

Figure 5. Comparison of numerical solutions for an advective 2D solute transport at $\mathrm{GPe}=50$ : (a) D2Q4 SRT, D2Q5 SRT, Crank-Nicolson (CN), and Explicit Finite Difference (EFD) and (b) D2Q5 MRT and D2Q9 SRT.

analytical solution, expressed by Eq. (27), does not work out for the above-mentioned advection-dominated problems owing to the infinite values of its exponential terms.

Therefore, it is crucial to determine a limitation for each LB model to prevent unavoidable artificial fluctuations in the numerical solutions. The bigger the GPN is, the more the advective mass transport problem will be. The oscillation rates of the LB and FD methods versus GPN are illustrated in Figures 7 and 8 , respectively. These figures have been obtained by several numerical tests at different values of velocity,

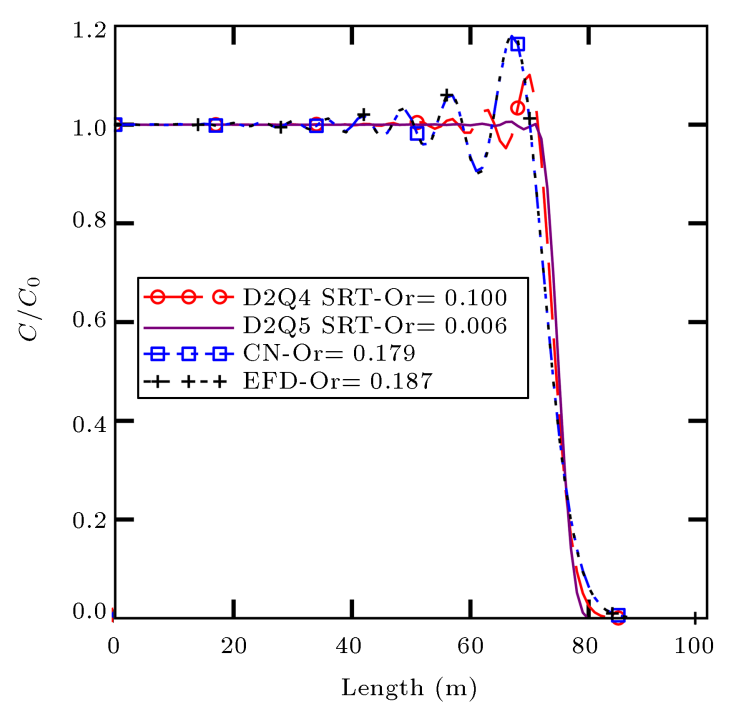

(a)

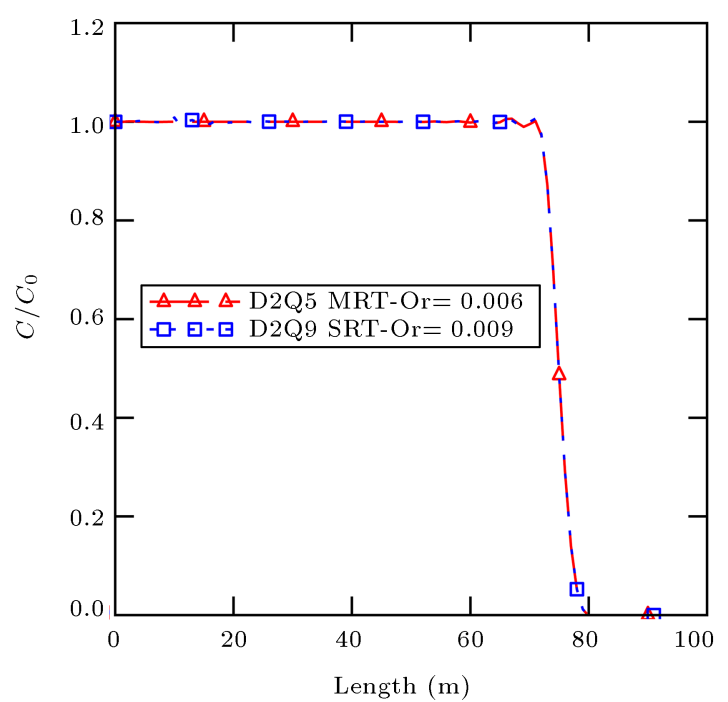

(b)

Figure 6. Comparison of numerical solutions for an advective $2 \mathrm{D}$ solute transport at $\mathrm{GPe}=50$ (length -concentration curve): (a) D2Q4 SRT, D2Q5 SRT, Crank-Nicolson (CN), and Explicit Finite Difference (EFD) and (b) D2Q5 MRT and D2Q9 SRT.

dispersion coefficient, and grid size. As shown in Figure 7, D2Q5 SRT and D2Q9 SRT meet the numerical oscillations nearly at GPN values greater than 25, D2Q5 MRT faces these oscillations when GPN is greater than 20, and both D2Q4 SRT and D2Q9 MRT confront the artificial over/undershoots once GPN is more than 10. However, the numerical fluctuations of EFD and CN methods begin as soon as GPN is 2 and 5, respectively (Figure 8). These results confirm that the LB methods are more accurate than the FD methods dealing with advective transport problems. More importantly, D2Q5 SRT and D2Q9 SRT, followed by D2Q5 MRT, are more powerful than D2Q4 SRT 


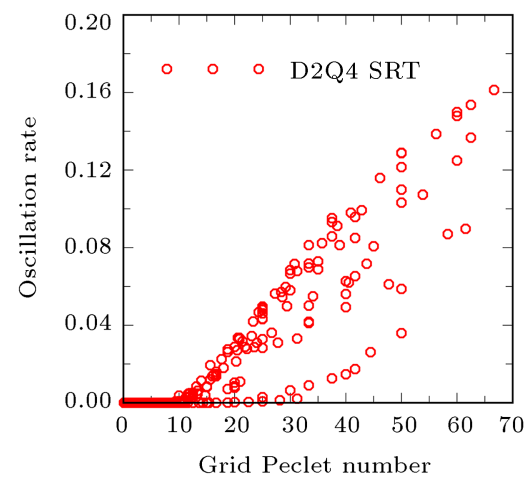

(a)

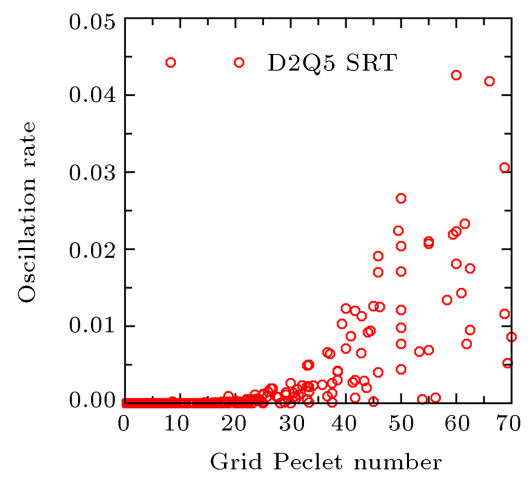

(b)

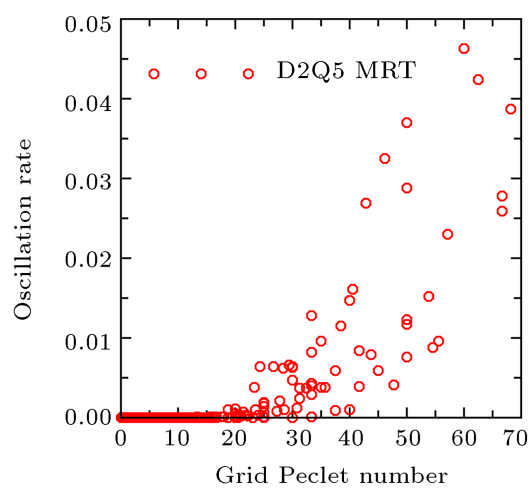

(c)

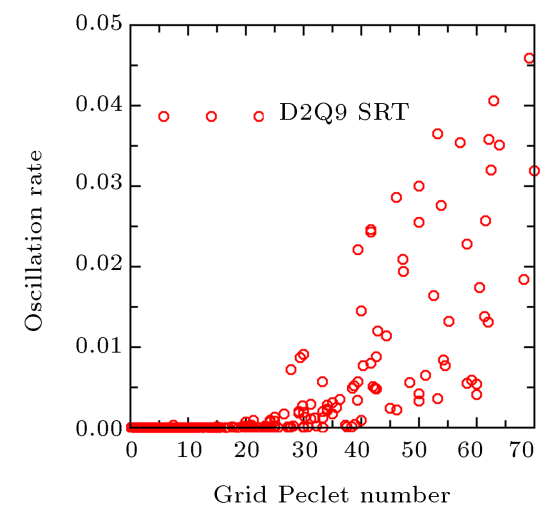

(d)

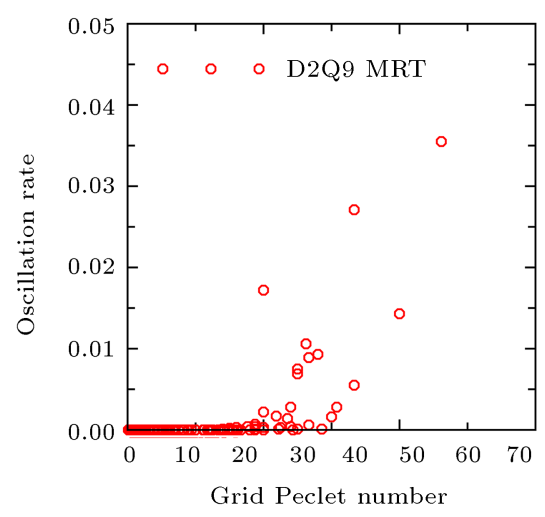

(e)

Figure 7. Oscillations of LB models: (a) D2Q4 SRT, (b) D2Q5 SRT, (c) D2Q5 MRT, (d) D2Q9 SRT, and (e) D2Q9 MRT.

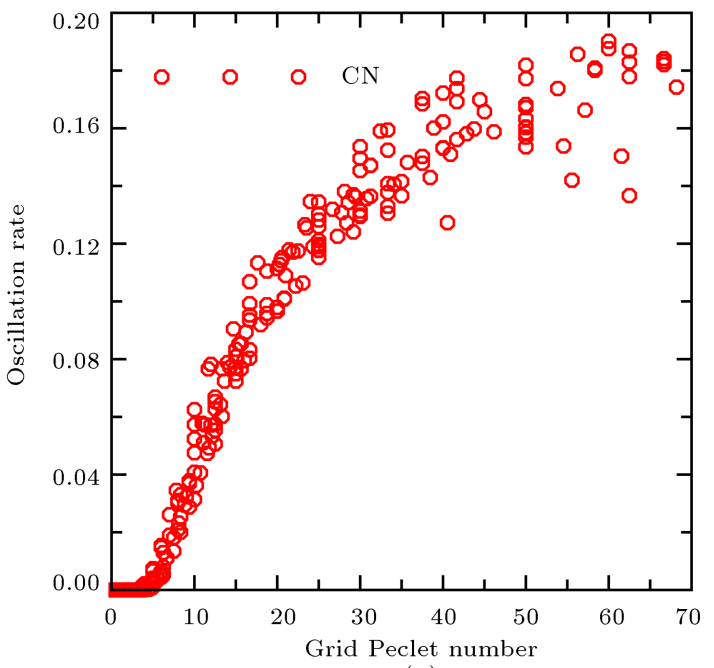

(a)

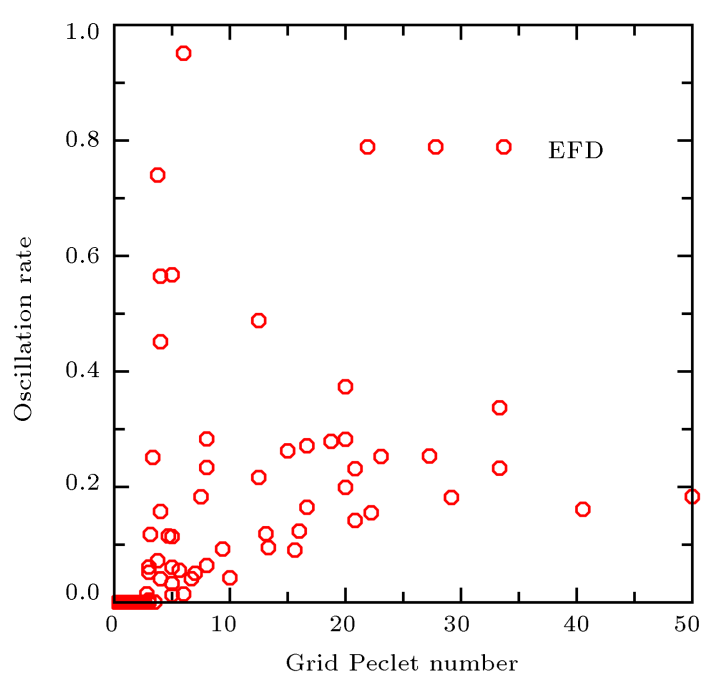

(b)

Figure 8. Oscillations of Finite Difference (FD) models: (a) Crank-Nicolson (CN) and (b) Explicit Finite Difference (EFD).

and D2Q9 MRT in modeling advection-controlled mass transport equations.

It is worth mentioning that there are small fluctuations at the early times of the time-concentration curve obtained from D2Q5 SRT (Figure 9(a)). However, these first oscillations disappear by the employ- ment of D2Q5 MRT (Figure 9(b)). This indicates that the application of MRTs provides a smoother solution, especially at the early times. It should be mentioned that stable solutions were taken into consideration in the above computations. The most important stability criterion for LB methods is to select 


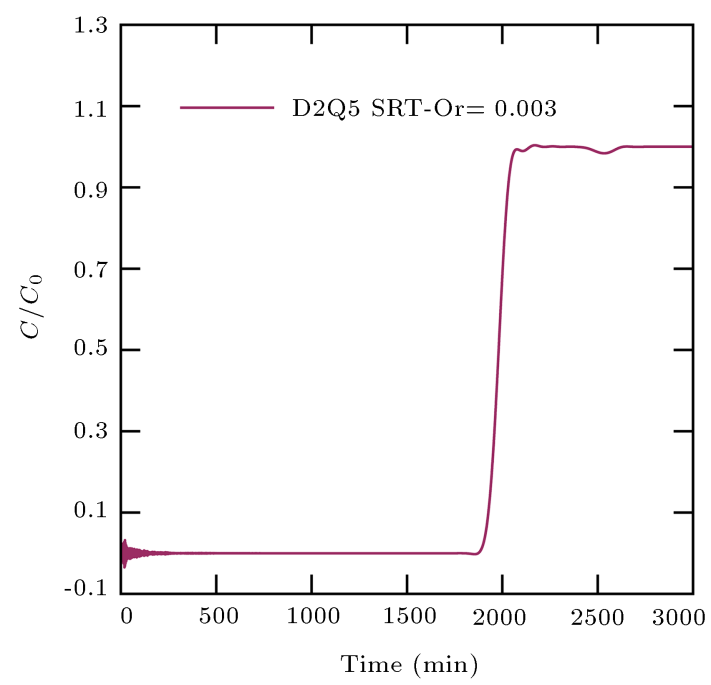

(a)

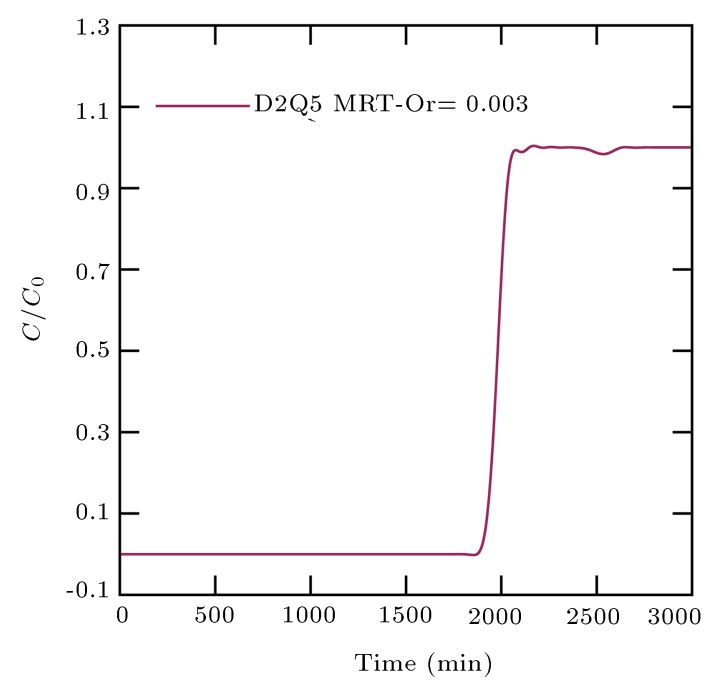

(b)

Figure 9. Effect of Single Relaxation Time (SRT) and Multiple Relaxation Time (MRT) on the solution: (a) D2Q5 SRT and (b) D2Q5 MRT.

a time step that satisfies $1 / \tau<2$ [18]. More stable conditions were also considered in the selection of time steps according to references [34-37]. Besides, the influence of time step on these numerical fluctuations was examined and measured using Courant number $(\mathrm{Cr}=u \cdot \Delta t / \Delta x)$. The variations of Oscillation Rate (Or) versus $\mathrm{Cr}$ and GPN for D2Q5 SRT are shown in Figure 10. It is observed that the OR is independent of Cr. Similar figures were attained for the other numerical schemes, which are not shown here for the sake of brevity.

The above results are based on the linear form of EDF, described by Eq. (7). Nonetheless, it is possible to use the EDF with more velocity terms as defined by Eq. (20). The numerical solutions of D2Q4 SRT, D2Q5 SRT, and D2Q9 SRT using both linear and quadratic

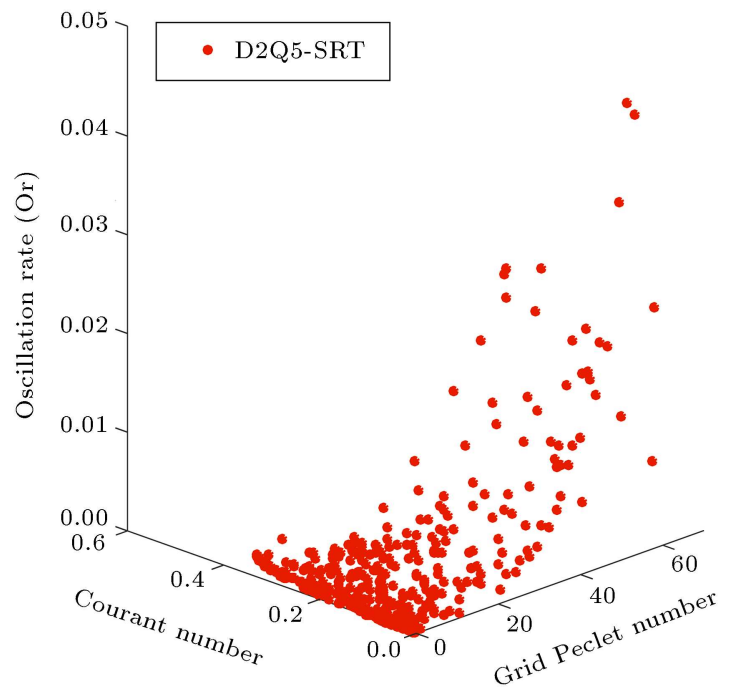

Figure 10. Effect of Courant number (Cr) and Grid Peclet Number (GPN) on artificial oscillations regarding D2Q5 SRT.

EDF for the advective mass transport problem are drawn in Figure 11. In this figure, the GPN is 100 . As shown, the magnitude of fluctuations is the same for both EDFs. Consequently, the employment of quadratic EDF causes no improvement in reducing the numerical non-natural over/undershoots.

\subsection{Numerical results for ordinary advection-dispersion equation}

In terms of an ordinary advection-dispersion equation, the numerical and analytical time-concentration curves are shown in Figure 12. Here, both advection and dispersion phenomena have significant roles in the mass transport. The model parameters are $u_{x}=0.05 \mathrm{~m} / \mathrm{min}$ and $D_{x}=D_{y}=0.05 \mathrm{~m}^{2} / \mathrm{min}$; the GPN is equal to 1 (Model 1 in Table 1). The LB models, as well as the CN and EFD methods, reveal very close agreement with the analytical solutions. The small values of RMSE, ranging from 0.57 to 1.22 , also confirm the close agreement between the numerical and analytical answers.

Based on the values of RMSE, it is deduced that D2Q5 MRT, D2Q4 SRT, and D2Q5 SRT lead to closer agreement with the analytical solution in comparison with D2Q9 SRT and D2Q9 MRT. This close correspondence between the predictions of LB methods and the analytical answers is observed in the plot of concentration contour lines. The concentration contour lines obtained by both D2Q5 SRT and analytical solutions are shown in Figure 13.

Even though the predictions of the abovementioned numerical methods are in good agreement with the analytical solutions, their computational times are different. Regarding the above ordinary advectiondispersion problem, the relative computational time 
Table 2. Relative computational time of Lattice Boltzmann (LB) and Finite Difference (FD) models with respect to Explicit Finite Difference (EFD) (advection-dispersion problem).

\begin{tabular}{|c|c|c|c|c|c|c|c|c|c|c|c|}
\hline \multirow[b]{2}{*}{ Model } & \multicolumn{4}{|c|}{ Model parameters } & \multicolumn{7}{|c|}{ Computational time } \\
\hline & $\begin{array}{c}u_{x} \\
(\mathrm{~m} / \mathrm{min})\end{array}$ & $\begin{array}{c}D_{x} \\
\left(\mathrm{~m}^{2} / \min \right)\end{array}$ & $\begin{array}{l}d x \\
(\mathbf{m})\end{array}$ & $\begin{array}{c}d t \\
(\mathrm{~min})\end{array}$ & EFD & $\mathrm{CN}$ & $\begin{array}{c}\text { D2Q4 } \\
\text { SRT }\end{array}$ & $\begin{array}{c}\text { D2Q5 } \\
\text { SRT }\end{array}$ & $\begin{array}{l}\text { D2Q5 } \\
\text { MRT }\end{array}$ & $\begin{array}{c}\text { D2Q9 } \\
\text { SRT }\end{array}$ & $\begin{array}{l}\text { D2Q9 } \\
\text { MRT }\end{array}$ \\
\hline Model A & 0.05 & 0.05 & 1 & 5 & 1 & 172 & 3.5 & 3.6 & 98 & 14.4 & 124 \\
\hline Model B & 0.05 & 0.05 & 1 & 12 & 1 & 283 & 3.1 & 3.5 & 95.5 & 14 & 107 \\
\hline
\end{tabular}

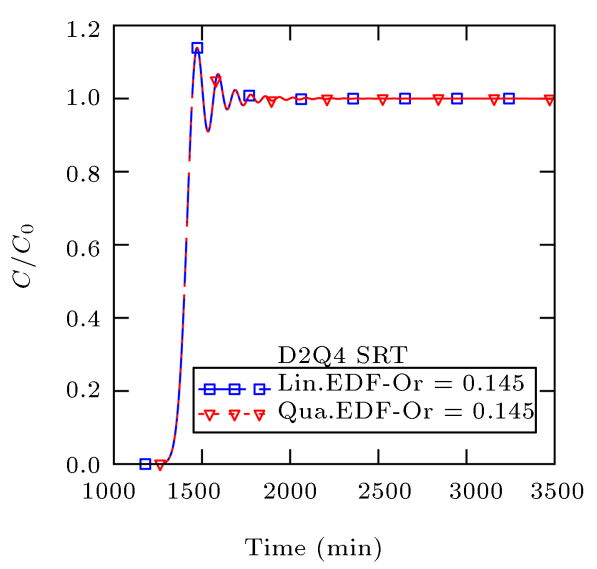

(a)

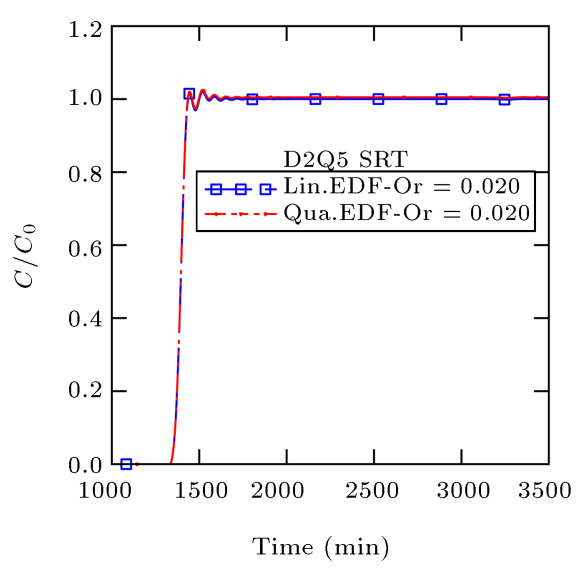

(b)

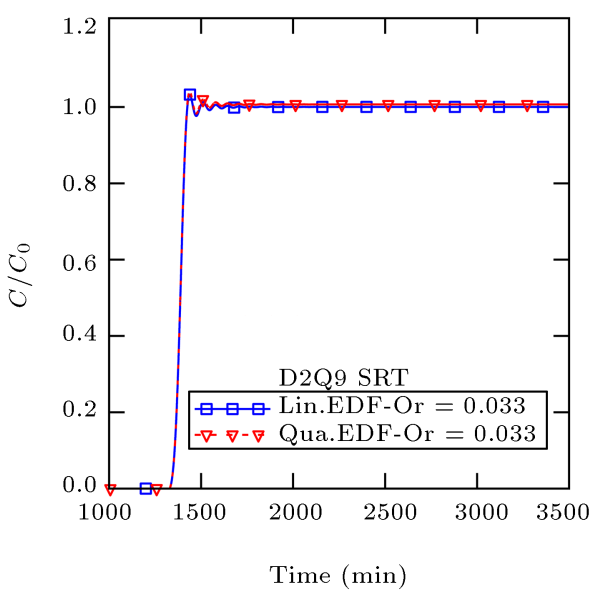

(c)

Figure 11. Influence of linear and quadratic EDFs: (a) D2Q4 SRT, (b) D2Q5 SRT, and (c) D2Q9 SRT.

of each scheme with respect to the EFD method is listed in Table 2. Two cases with different time steps (models A and B) were taken into consideration. Accordingly, all LB models are much faster than CN method, because CN method is an implicit scheme that requires matrix inversion in each time step. It is also observed that the computational speeds of SRT schemes, including D2Q4 SRT and D2Q5 SRT, are much faster than those of MRT schemes as a result of matrix operation in the MRT models. For instance, D2Q5 SRT scheme is over 25 times faster than D2Q5 MRT scheme.

\subsection{Numerical results of coupled groundwater and solute transport equations}

The seepage flow and the solute transport underneath the recharge dam, as explained in Figure 3, were modeled simultaneously using all LB and FD models. The equipotential lines and the flow net, attained by D2Q5 SRT and CN schemes, are depicted in Figure 14. As shown, there is close agreement between these two numerical models, indicating an equal accuracy rate of D2Q5 SRT and CN schemes in the description of seepage flow beneath the recharge dam pond. Similar agreements are also observed for the other aforesaid 


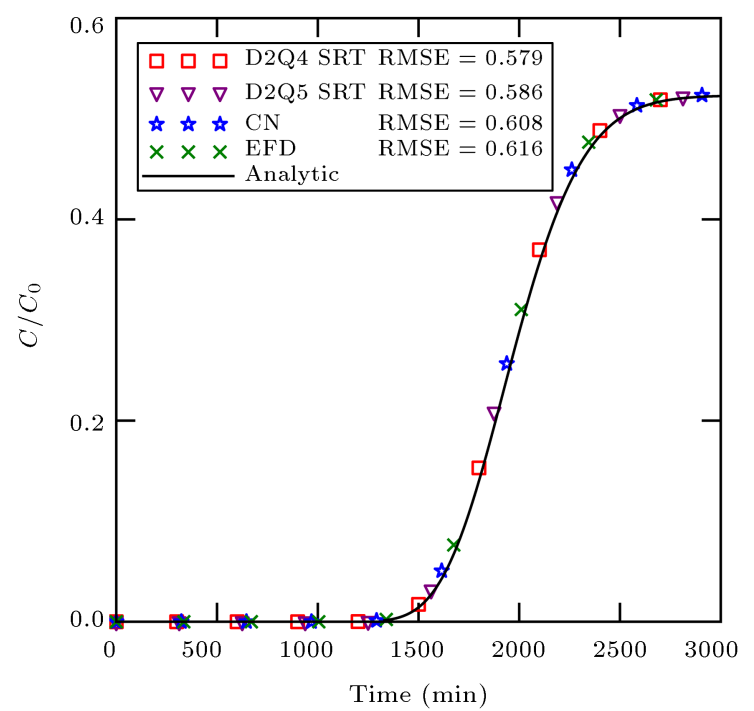

(a)

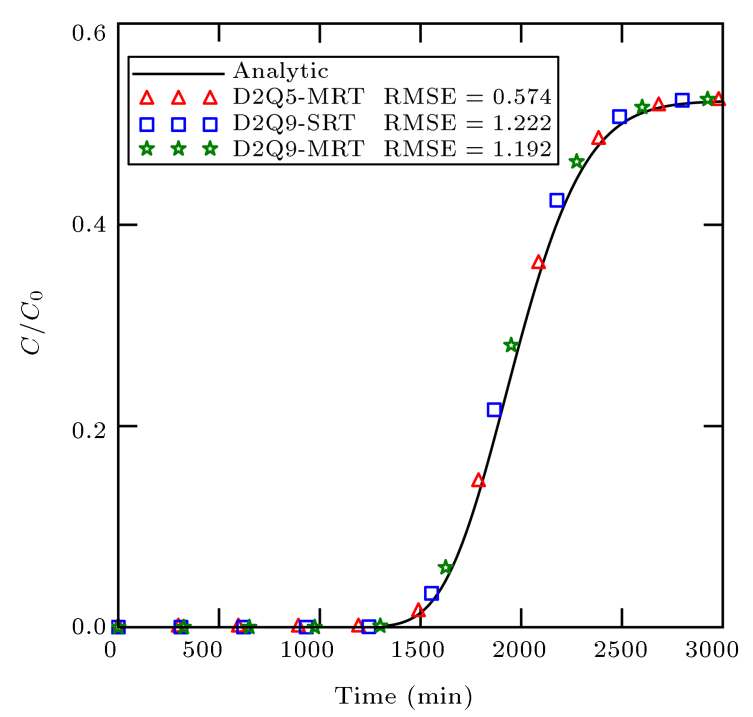

(b)

Figure 12. Comparison of numerical solutions concerning an ordinary $2 \mathrm{D}$ solute transport at $\mathrm{GPe}=1$ : (a) D2Q4 SRT, D2Q5 SRT, Crank-Nicolson (CN), and Explicit Finite Difference (EFD) and (b) D2Q5 MRT, D2Q9 SRT, and D2Q9 MRT.

LB schemes, which are not shown here for the sake of brevity.

Figure 15 displays the time-concentration curves associated with different LB and FD methods at the point " $w$ " in Figure 3. As shown, the results of D2Q4 SRT, D2Q5 SRT, D2Q5 MRT, D2Q9 SRT, and D2Q9 MRT models, in addition to EFD and CN schemes, are in high accordance. It is also shown that LB models are capable of dealing with a combination of different boundary conditions, comprising Dirichlet, Neumann, and open boundary conditions. Interestingly, the computational durations of the LB and FD models

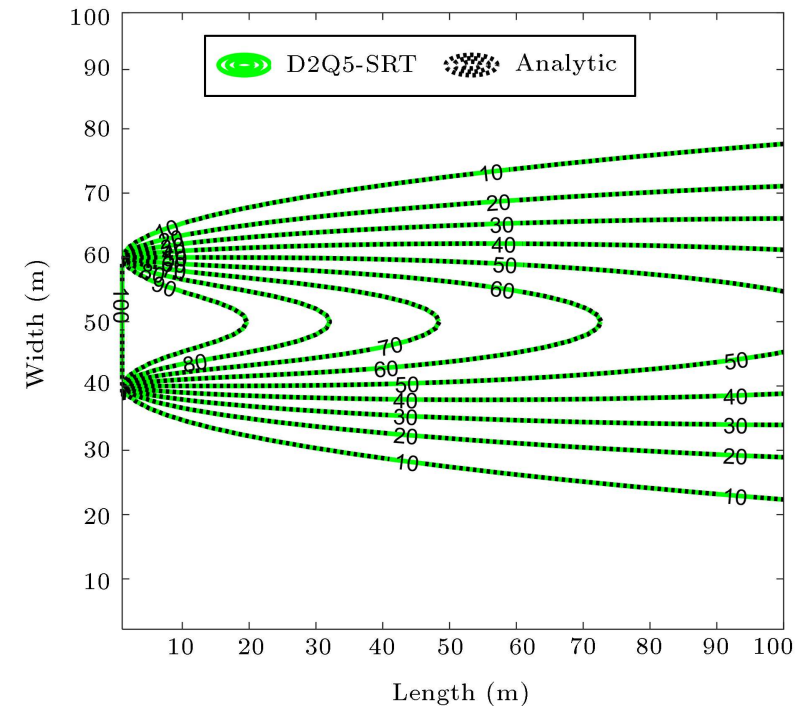

Figure 13. Solute concentration contour lines.

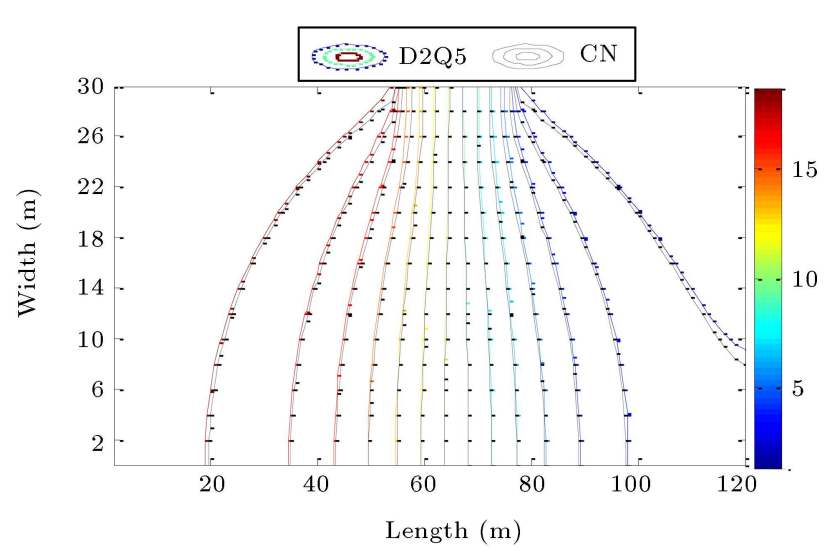

(a)

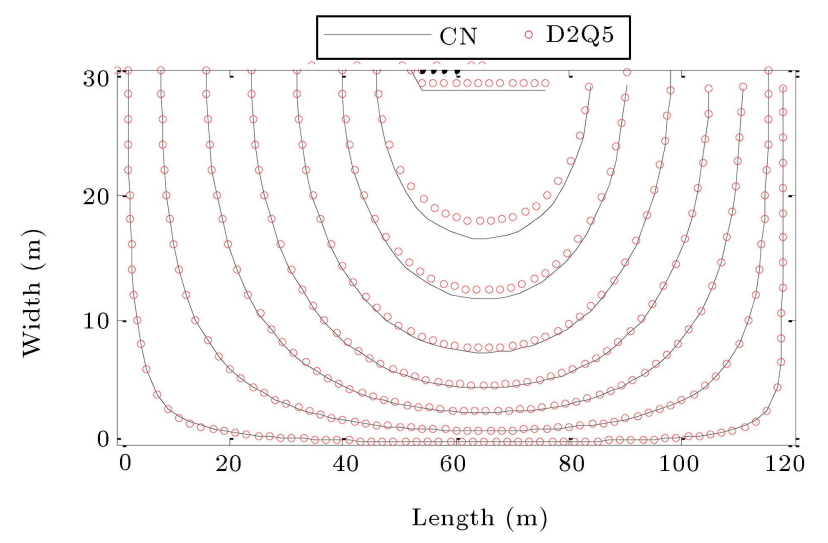

(b)

Figure 14. Comparison of D2Q5 and Crank-Nicolson (CN) methods: (a) Equipotential lines and (b) streamlines.

were completely different (Table 3 ). Among the LB methods, D2Q4-SRT, followed by D2Q5-SRT, gave rise to the highest computational speed, which is close to the speed of EFD method. Similar to the results of ordinary solute transport problem, as explained earlier 


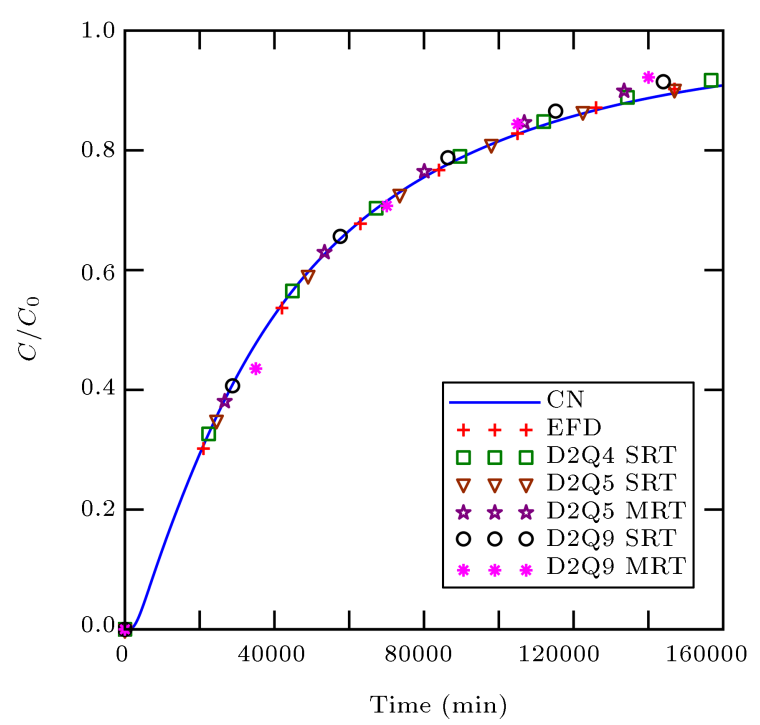

Figure 15. Time-concentration curve resulting from the Lattice Boltzmann (LB) and Finite Difference (FD) models in a coupled system of groundwater and solute transport problem.

Table 3. Relative computational time of Lattice Boltzmann (LB) and Finite Difference (FD) models with respect to Explicit Finite Difference (EFD) (coupled system).

\begin{tabular}{ccccccc}
\hline EFD & CN & $\begin{array}{c}\text { D2Q4 } \\
\text { SRT }\end{array}$ & $\begin{array}{c}\text { D2Q5 } \\
\text { SRT }\end{array}$ & $\begin{array}{c}\text { D2Q5 } \\
\text { MRT }\end{array}$ & $\begin{array}{c}\text { D2Q9 } \\
\text { SRT }\end{array}$ & $\begin{array}{c}\text { D2Q9 } \\
\text { MRT }\end{array}$ \\
\hline 1 & 56 & 1.1 & 1.1 & 11.3 & 3.8 & 14 \\
\hline
\end{tabular}

(Table 2), the computational speed of SRT schemes is much faster than that of MRT schemes.

\section{Conclusion}

In this study, five schemes of Lattice Boltzmann (LB) methods were employed to solve both ordinary and advection-dominated solute transport problems. Furthermore, the solutions of LB models were compared with those of Finite Difference (FD) models. It is deduced that LB models face artificial oscillations at greater Grid Peclet Number (GPN) values in comparison with the FD schemes, indicating the high efficiency of LB models to describe the advective mass transport problems.

Amongst the LB models, D2Q5 SRT and D2Q9 SRT, followed by D2Q5 MRT, are more accurate than D2Q4 SRT and D2Q9 MRT. The aforementioned LB models, comprising D2Q4 SRT, D2Q5 SRT, D2Q5 MRT, D2Q9 SRT, and D2Q9 MRT, face numerical over/undershoots when GPN is greater than 10, 25, 20, 25, and 10, respectively. Nonetheless, the Explicit Finite Difference (EFD) and Crank-Nicolson (CN) methods start synthetic oscillations at GPNs of 2 and 5, respectively. Besides, small fluctuations were perceived at the early times of the time-concentration curve attained by D2Q5 SRT model, while these fluctuations disappear by the application of Multiple Relaxation Time (MRT) in lieu of Single Relaxation Time (SRT). In addition, the employment of quadratic Equilibrium Distribution Function (EDF) instead of the linear one leads to the same magnitude of artificial oscillations in the LB solutions.

Considering the ordinary advection-dispersion equation, D2Q5 MRT, D2Q4 SRT, and D2Q5 SRT result in more accurate solutions in comparison with those of D2Q9 SRT and D2Q9 MRT. Finally, by considering a recharge dam pond, a combination of groundwater flow and solute transport was solved successfully using LB methods. Although the LB models ended up with identical solutions, the computational speeds of SRT models were found to be faster than MRT models. Besides, all LB models were much faster than CN method.

\section{Acknowledgments}

The authors would like to thank Shiraz University of Technology for supporting this research.

\section{References}

1. Amiri, S., Talebbeydokhti, N., and Baghlani, A. "A two-dimensional well-balanced numerical model for shallow water equations", Sci. Iran., 20(1), pp. 97-107 (2013).

2. Mahdavi, A. and Talebbeydokhti, N. "A hybrid solid boundary treatment algorithm for smoothed particle hydrodynamics", Sci. Iran., 22(4), pp. 1457-1469 (2015).

3. Asadollahfardi, G., Rezaee, M., and Mehrjardi, G.T. "Simulation of unenhanced electrokinetic process for lead removal from kaolinite clay", Int. J. Civ. Eng., 14(4), pp. 263-270 (2016).

4. Alemi, M. and Maia, R. "Numerical simulation of the flow and local scour process around single and complex bridge piers", Int. J. Civ. Eng., 16(5), pp. 475-487 (2018).

5. Hekmatzadeh, A.A., Papari, S., and Amiri, S.M. "Investigation of energy dissipation on various configurations of stepped spillways considering several RANS turbulence models", Ijst-T. Civ. Eng., 42(2), pp. 97109 (2018).

6. Mohamad, A.A. and Kuzmin, A. "A critical evaluation of force term in lattice Boltzmann method, natural convection problem", Int. J. Heat Mass Transfer, 53(5), pp. 990-996 (2010).

7. Perumal, D.A. and Dass, A.K. "A review on the development of lattice Boltzmann computation of macro fluid flows and heat transfer", Alexandria Eng. J., 54(4), pp. 955-971 (2015). 
8. Yan, Z., Yang, X., Li, S., and Hilpert M. "Tworelaxation-time lattice Boltzmann method and its application to advective-diffusive-reactive transport", Adv. Water Resour., 109, pp. 333-342 (2017).

9. Guo, Z. and Shu, C., Lattice Boltzmann Method and Its Applications in Engineering, World Scientific, Singapore (2013).

10. Gao, J., Xing, H., Tian, Z., Pearce, J.K., Sedek, M., and Golding, S.D. "Reactive transport in porous media for $\mathrm{CO} 2$ sequestration: Pore scale modeling using the lattice Boltzmann method", Comput. Geosci., 98, pp. 9-20 (2017).

11. Yang, Y. and Wang, M. "Pore-scale modeling of chloride ion diffusion in cement microstructures", Cement Concrete Comp., 85, pp. 92-104 (2018).

12. Shi, B. and Guo, Z. "Lattice Boltzmann simulation of some nonlinear convection-diffusion equations", Comput. Math. Appl., 61(12), pp. 3443-3452 (2011).

13. Sharma, K.V., Straka, R., and Tavares, F.W. "New cascaded thermal lattice Boltzmann method for simulations of advection-diffusion and convective heat transfer", Int. J. Therm. Sci., 118, pp. 259-277 (2017).

14. Wang, H., Cater, J., Liu, H., Ding, X., and Huang, W. "A lattice Boltzmann model for solute transport in open channel flow", J. HYDROL., 556, pp. 419-426 (2018).

15. Mohamad, A.A., Lattice Boltzmann Method: Fundamentals and Engineering Applications with Computer Codes, Springer Science \& Business Media, Springer (2011).

16. Ibrahem, A.M., El-Amin, M.F., and Mohammadein, A.A. "Lattice Boltzmann technique for heat transport phenomena coupled with melting process", Heat Mass Transfer, 53(1), pp. 213-221 (2017).

17. Xia, Y., Wu, J., and Zhang, Y. "Lattice-Boltzmann simulation of two-dimensional super-diffusion", Eng. Appl. Comp. Fluid., 6(4), pp. 581-594 (2012).

18. Zhou, J.G. "A lattice Boltzmann method for solute transport", Int. J. Numer. Methods Fluids, 61(8), pp. 848-863 (2009).

19. Yoshida, H. and Nagaoka, M. "Lattice Boltzmann method for the convection-diffusion equation in curvilinear coordinate systems", J. Comput. Phys., 257, pp. 884-900 (2014).

20. Perko, J. and Patel, R.A. "Single-relaxation-time lattice Boltzmann scheme for advection-diffusion problems with large diffusion-coefficient heterogeneities and high-advection transport", Phys. Rev. E, 89(5), p. 053309 (2014).

21. Hosseini, R., Rashidi, S., and Esfahani, J.A. "A lattice Boltzmann method to simulate combined radiationforce convection heat transfer mode", J. Braz. Soc. Mech. Sci. Eng., 2017, pp. 1-12 (2017).

22. Zheng, Y., Li, G., Guo, W., and Dong, C. "Lattice Boltzmann simulation to laminar pulsating flow past a circular cylinder with constant temperature", Heat Mass Transfer, 2017, pp. 1-12 (2017).

23. Bin, D., Bao-Chang, S., and Guang-Chao, W. "A new lattice Bhatnagar-Gross-Krook model for the convection-diffusion equation with a source term", Chin. Phys. Lett., 22(2), pp. 267-270 (2005).

24. Batot, G., Talon, L., Peysson, Y., Fleury, M., and Bauer, D. "Analytical and numerical investigation of the advective and dispersive transport in HerschelBulkley fluids by means of a lattice-Boltzmann tworelaxation-time scheme", Chem. Eng. Sci., 141, pp. 271-281 (2016).

25. Huang, R. and $\mathrm{Wu}, \mathrm{H}$. "A modified multiplerelaxation-time lattice Boltzmann model for convection-diffusion equation", J. Comput. Phys., 274, pp. 50-63 (2014).

26. Li, L., Mei, R., and Klausner, J.F. "Lattice Boltzmann models for the convection-diffusion equation: D2Q5 vs D2Q9", Int. J. Heat Mass Transfer, 108, pp. 41-62 (2017).

27. Chai, Z. and Zhao, T. "Nonequilibrium scheme for computing the flux of the convection-diffusion equation in the framework of the lattice Boltzmann method", Phys. Rev. E, 90(1), p. 013305 (2014).

28. Liu, Q., He, Y.L., Li, Q., and Tao, W.Q. "A multiplerelaxation-time lattice Boltzmann model for convection heat transfer in porous media", Int. J. Heat Mass Transfer, 73, pp. 761-775 (2014).

29. Chopard, B., Falcone, J., and Latt, J. "The lattice Boltzmann advection-diffusion model revisited", Eur. Phys. J. Special Topics, 171(1), pp. 245-249 (2009).

30. Li, Q., Chai, Z., and Shi, B. "Lattice Boltzmann model for a class of convection-diffusion equations with variable coefficients", Comput. Math. Appl., 70(4), pp. 548-561 (2015).

31. Hekmatzadeh, A.A., Karimi-Jashani, A., Talebbeydokhti, N., and Kløve, B. "Modeling of nitrate removal for ion exchange resin in batch and fixed bed experiments", Desalination, 284, pp. 22-31 (2012).

32. Zheng, C. and Bennett, G.D., Applied Contaminant Transport Modeling, Wiley-Interscience, New York (2002).

33. Krivovichev, G.V. "Numerical stability analysis of lattice Boltzmann equations for linear diffusion", Appl. Math. Inf. Sci., 9(4), pp. 1687-1692 (2014).

34. Niu, X., Shu, C., Chew, Y.T., and Wang, T.G. "Investigation of stability and hydrodynamics of different lattice Boltzmann models", J. Stat. Phys., 117(3), pp. 665-680 (2004).

35. Servan-Camas, B. and Tsai, F.T.C. "Non-negativity and stability analyses of lattice Boltzmann method for advection-diffusion equation", J. Comput. Phys., 228(1), pp. 236-256 (2009).

36. Sterling, H.D. and Chen, S. "Stability analysis of lattice Boltzmann methods", J. Comput. Phys., 123(1), pp. 196-206 (1996). 
37. Suga, S. "Numerical schemes obtained from lattice Boltzmann equations for advection diffusion equations", Int. J. Mod. Phys. C, 17(11), pp. 1563-1577 (2006).

38. Rao, P.R. and Schaefer, L.A. "Numerical stability of explicit off-lattice Boltzmann schemes: A comparative study", J. Comput. Phys., 285, pp. 251-264 (2015).

39. Huang, H., Lu, X., and Sukop, M. "Numerical study of lattice Boltzmann methods for a convection-diffusion equation coupled with Navier-Stokes equations", $J$. Phys. A: Math. Theor., 44(5), p. 055001 (2011).

40. Li, L., Mei, R., and Klausner, J.F. "Multiplerelaxation-time lattice Boltzmann model for the axisymmetric convection diffusion equation", Int. J. Heat Mass Transfer, 67, pp. 338-351 (2013).

41. Liu, H., Zhou, J.G., Li, M., and Zhao, Y. "Multi-block lattice Boltzmann simulations of solute transport in shallow water flows", Adv. Water Resour., 58, pp. 2440 (2013).

42. Seta, T., Takegoshi, E., and Okui, E. "Lattice Boltzmann simulation of natural convection in porous media", Math. Comput. Simul., 72(2), pp. 195-200 (2006).

43. Karamouz, M., Ahmadi, A., and Akhbari, M., Groundwater Hydrology: Engineering, Planning, and Management, CRC Press, Boca Raton (2011).

44. Wang, H. and Anderson, M., Introduction to Groundwater Modeling: Finite Differences and Finite Element Methods, Freeman, San Francisco (1982).

45. Guo, Z. and Shu, C., Lattice Boltzmann Method and Its Applications in Engineering, World Scientific, Singapore (2013).

46. Saadat, S., Hekmatzadeh, A.A., and Karimi-Jashni, A. "Mathematical modeling of the Ni (II) removal from aqueous solutions onto pre-treated rice husk in fixedbed columns: a comparison", Desalin. Water Treat., 57(36), pp. 16907-16918 (2016).

47. Reilly, T.E., Franke, L., and Bennett, G.D. "The principle of superposition and its application in groundwater hydraulics", Techniques of Water-Resources Investigations of the United States Geological Survey (1984).

48. Al-Turbak, A.S. and Al-Muttair, F.F. "Evaluation of dams as a recharge method", Int. J. Water Resour. Dev., 5(2), pp. 119-124 (1989).

49. Abdalla, O.A. and Al-Rawahi, A.S. "Groundwater recharge dams in arid areas as tools for aquifer replenishment and mitigating seawater intrusion: example of AlKhod, Oman", Environ. Earth. Sci., 69(6), pp. 1951-1962 (2013).

\section{Biographies}

Ali Akbar Hekmatzadeh is currently an Assistant Professor at the Department of Civil and Environmental Engineering in Shiraz University of Technology, Shiraz, Iran since 2014. He received BSc, MSc, and PhD degrees, all in Civil and Environmental Engineering from Shiraz University, Shiraz, Iran. His areas of research and interest are groundwater modeling and pollution transport, hydraulic structures, water treatment processes, Computational Fluid Dynamics (CFD), and reliability in engineering problems and hydrology.

Hatef Keshavarzi received a BS degree in Civil Engineering from Sirjan University of Technology, Sirjan, Iran in 2014 and an MS degree in Water Engineering and Hydraulic Structures in 2017 from Shiraz University of Technology, Iran. His research interests include numerical modeling of groundwater and pollution transport and hydraulic structures.

Nasser Talebbeydokhti is a Professor of Civil and Environmental Engineering from Shiraz University, Shiraz, Iran since 1984. He is currently a member of Academy of Science and the editor-in-chief of Iranian Journal of Science and Technology Transaction of Civil Engineering. In recognition of his works and significant contributions, he was the recipient of the 2017 nationwide distinguished professor at Ministry of Higher Education for demonstrated excellence in the areas of teaching, research, instruction, university service, and public service. He was also the recipient of the Distinguished Hydraulic Professor by Iran Hydraulic Society.

Ali Torabi Haghighi is a lecturer and a post-doctoral researcher at University of Oulu. He holds a PhD degree on Water Resources \& Geo-environmental Engineering from University of Oulu, Finland (2010-2014). His previous positions include visiting Researcher in University of California, USA, manager of dam and irrigation projects, monitoring and operation of dams in Fars Regional Water Authority, Shiraz, Iran (19982009). His research interests lie generally in the areas of water resources and more specifically in the study of Lake and river flow regime, large-scale hydrology, cold climate hydrology, environmental flow assessment, political and socio-economic aspects of water resource systems, sustainable water resources development, extreme hydrological events, river engineering, remote sensing in water resources, dam and hydropower operation, and geotechnical engineering. 